\title{
Microwave absorption saturation and decay heating of surface electrons on liquid helium
}

\author{
Yuriy Monarkha \\ B. Verkin Institute for Low Temperature Physics and Engineering of the National Academy of Sciences of Ukraine \\ 47 Lenin Ave., Kharkov 61103, Ukraine \\ E-mail: monarkha@ilt.kharkov.ua
}

Denis Konstantinov and Kimitoshi Kono

Low Temperature Physics Laboratory, RIKEN, Hirosawa 2-1, Wako 351-0198, Japan

Received March 28, 2007

\begin{abstract}
The microwave (MW) resonance absorption and decay heating of surface electrons (SEs) on liquid ${ }^{4} \mathrm{He}$ are theoretically studied for the vapor atom scattering regime. The decay heating is shown to be an essential occurrence of a MW resonance experiment appearing even at low excitation rates. It strongly affects the occupancies of surface levels and the broadening of resonance lines long before the absorption suturation condition is reached. Contrary to the model of cold SEs usually used for description of the MW resonance, the new theory leads to MW absorption saturation when only a very small fraction of electrons (less than $10 \%$ ) is left on the ground and the first excited levels.
\end{abstract}

PACS: $67.90 .+\mathbf{z}$ Other topics in quantum fluids and solids; liquid and solid helium;

73.20. $-\mathbf{r}$ Electron states at surfaces and interfaces;

73.25. $+\mathbf{i}$ Surface conductivity and carrier phenomena;

78.70.Gq Microwave and radio-frequency interactions.

Keywords: surface state electrons on liquid helium, Rydberg states, microwave absorption, power broadening.

The microwave (MW) resonance absorption experiment [1] had given the first direct observation of image-potential-induced Rydberg levels outside liquid helium. For weak holding fields $E_{\perp}$, positions of surface levels were shown to be well described by the simple formula $\Delta_{l}=-\Delta_{R} / l^{2}$, where $l=1,2, \ldots$, and $\Delta_{R}$ is the corresponding Rydberg energy which is about $7.6 \mathrm{~K}$ for liquid ${ }^{4} \mathrm{He}$. Because the dielectric constant of liquid helium $\varepsilon$ is close to unity $(\varepsilon-1 \simeq 0.057)$, the effective Bohr radius of these Rydberg levels, $b=4\left(\hbar^{2} / m e^{2}\right)(\varepsilon+1) /(\varepsilon-1)$, is large (about $76 \AA$ ). Therefore, surface electrons (SEs) can move freely along the interface, forming a remarkable two-dimensional (2D) electron liquid, useful for studying many-body effects in a highly correlated $2 \mathrm{D}$ electron gas (for a review see [2]).

The recent interest in the MW resonance is evoked by experimental development of the idea of using SEs as electronic qubits (see [3] and references there in). These qubits would be controlled by the MW field whose frequency $\omega$ is close to $\omega_{0}=\left(\Delta_{2}-\Delta_{1}\right) / \hbar$ (usually, the sys- tem is tuned to resonance by varying $\left.E_{\perp}\right)$. Since the electron potential is anharmonic, it is assumed that coupling to outer levels $(l>2)$ is negligible, and a simple two-level model is an excellent approximation. As an important achievement in this field, observation of MW absorption saturation of Rydberg states of SEs was reported [3].

The electron decay from the excited surface level to the ground level due to scattering by vapor atoms and ripplons is a negative factor for SE qubits. MW absorption saturation appears when the stimulated absorption (emission) rate $r$ is larger than the decay rate of the first excited level $v_{2 \rightarrow 1} \equiv 1 / \tau$. The dimensionless parameter $r \tau$ could be considered as a measure of electron excitation by MW. In the simple model of cold SEs [3], for low excitation $r \tau<<1$, the fractional occupancy of the first excited level $n_{2}$ is much smaller than that of the ground level $n_{1}$. In this case, power absorbed by electrons from the filed $P_{\mathrm{A}}$ increases linear with $r$, or with the in-put power $P_{\text {in }}$. In the opposite limit of high excitation $r \tau \gg>1$, $n_{2} \rightarrow n_{1} \rightarrow 1 / 2$ and $P_{\mathrm{A}}=\left(n_{1}-n_{2}\right) \hbar \omega_{0} r$ saturates, while 
absorption lines become substantially affected by power broadening.

The above mentioned results were based on the key assumption that electron temperature $T_{e}$ remains to be close to the ambient temperature $T$ as the excitation parameter $r \tau$ increases. This was not proven for experimental conditions of Ref. 3. Moreover, already in 1980, there was an evidence supported by a simple estimate that MW can substantially heat SEs [4] at the resonance condition. In the important temperature range, electron scattering by vapor atoms and ripplons is accompanied by a very small energy exchange. When decaying back to the ground level an electron acquires huge kinetic energy of the in-plane motion, $\varepsilon_{k}=\hbar^{2} k^{2} / 2 m \sim \Delta_{R}>T$, and decaying becomes a sort of shooting electrons along the surface. High electron-electron collisions quickly redistribute this kinetic energy among other electrons, which should lead to decay heating of SEs. Therefore, an accurate analysis of MW resonance absorption of decay-heated SEs is highly desirable.

In this work we report the results of theoretical description of MW resonance of SEs on liquid helium for the conditions of the experiment [3]. Electron temperature is found from the energy balance equation. We show that the decay heating cannot be disregarded even for rather low excitations $r \tau \sim 10^{-3}$, and, under the saturation condition $r \tau>1$, electron temperature more than 40 times (!) exceeds the ambient temperature. As a result, the limiting fraction of electrons left on the ground and the first excited levels which provides the decay heating of the rest electrons is very small (less than $10 \%$ ).

For vapor atom scattering regime, the excitation rate $r$ has its usual Lorentzian form [5]:

$$
r=\frac{1}{2} \frac{\Omega_{R}^{2} \gamma}{\delta^{2}+\gamma^{2}},
$$

where $\delta=\omega-\omega_{0}, \gamma$ is the linewidth, $\Omega_{R}=e E_{0}\langle 1|z| 2\rangle / \hbar$ is the Rabi frequency, $E_{0}$ is the MW field amplitude, and $\langle 1|z| 2\rangle$ is the electric dipole length for the transition. The line shape of power absorption $P_{\mathrm{A}}(\delta)$ coincides with that of $r(\delta)$, when $n_{1} \approx 1>n_{2}$. If $n_{1}$ is substantially reduced by excitation, the line-shape of $P_{\mathrm{A}}(\delta)$ will be changed.

Fractional occupancies $n_{l}$ should satisfy the rate equations for all surface levels. In the simple model of cold SEs [3], coupling to outer levels is disregarded, $n_{2}=r \tau /(1+2 r \tau)$, and, therefore,

$$
P_{\mathrm{A}}=\frac{\Delta_{21}}{\tau} \frac{1}{(r \tau)^{-1}+2},
$$

where $\Delta_{l^{\prime} l}=\Delta_{l^{\prime}}-\Delta_{l}$. At $r \tau>1, P_{\mathrm{A}}$ saturates, while the line-shape $P_{\mathrm{A}}(\delta)$ acquires a substantial broadening proportional to $\Omega_{R}^{2}$ (power broadening).
This simple picture changes drastically, if we do not fix electron temperature to $T$. The energy absorbed from the MW field is eventually transferred to vapor atoms. Therefore, electron temperature $T_{e}$ as a function of $r \tau$ is determined by the energy balance equation

$$
\left(n_{1}-n_{2}\right) \Delta_{21} r \tau=\left(T_{e}-T\right) \widetilde{v}\left(T_{e}\right) \tau,
$$

where $\widetilde{v}\left(T_{e}\right)$ is the energy relaxation rate. Because the electron mass $(m)$ is much smaller than the vapor atom mass $(M), \widetilde{v}(T) \tau$ is estimated to be about $10^{-3}$. Equation (3) makes decay heating obvious even by a simple estimate: if $\widetilde{v} \tau<<1$ and $n_{1}-n_{2} \sim 1$, at $r \tau \sim 1$, electron temperature $T_{e}$ should be much larger than $\Delta_{21}$. In other words, $T_{e}$ should be high enough to compensate the lack of electron energy relaxation.

For electron scattering by helium vapor atoms, the energy relaxation rate is found as [7]

$$
\begin{aligned}
\widetilde{v}= & \frac{m}{M} v_{a}^{(0)} \sum_{l, l^{\prime}} n_{l} \exp \left[-\left(\left|\Delta_{l^{\prime} l}\right|+\Delta_{l^{\prime} l}\right) / 2 T_{e}\right] \times \\
& \times\left[\left(\Delta_{R} / T_{e}\right) u_{l^{\prime} l}+\left(\left|\Delta_{l^{\prime} l}\right| / T_{e}+2\right) s_{l^{\prime} l}\right],
\end{aligned}
$$

where $v_{a}^{(0)}$ is the momentum relaxation rate for electron scattering within the ground level [6],

$$
\begin{gathered}
s_{l^{\prime} l}=\frac{B_{11}}{B_{l^{\prime} l}}, \quad B_{l^{\prime} l}^{-1}=\int_{0}^{\infty} d z\left[\chi_{l}(z) \chi_{l^{\prime}}(z)\right]^{2}, \\
u_{l^{\prime} l}=\frac{b^{2} B_{11}}{C_{l^{\prime} l}}, C_{l^{\prime} l}^{-1}=\int_{0}^{\infty} d z\left\{\frac{d}{d z}\left[\chi_{l}(z) \chi_{l^{\prime}}(z)\right]\right\}^{2},
\end{gathered}
$$

$\Delta_{R}=\hbar^{2} / 2 m b^{2}$, and $\chi_{l}(z)$ is the SE wave function. Usually, it is assumed that fractional occupancies $n_{l}$ are given by the simple Boltzmann distribution with an effective $T_{e}$. In this case, Eq. (4) gives the same energy relaxation rate as that found previously in the theory of nonlinear conductivity of hot SEs [6]. As we shall see below, this approximation is not sufficient for description of MW absorption saturation. At least for two lowest levels, the theory should be extended beyond the Boltzmann approximation.

The rate equations $d n_{m} / d t=0$ (here $m=1$ and 2) consist of the terms for transitions between the two lowest levels induced by the MW field $\left(n_{m^{\prime}}-n_{m}\right) r$, and terms for transitions between all levels due to scattering by vapor atoms. The rates for latter transitions are given by

$$
w_{l \rightarrow l^{\prime}}=v_{a}^{(0)} s_{l l^{\prime}} n_{l} \exp \left[-\left(\left|\Delta_{l^{\prime} l}\right|+\Delta_{l^{\prime} l}\right) / 2 T_{e}\right]
$$

[Eq. (5) defines also the life-time $\tau=1 / v_{a}^{(0)} s_{21}$ which does not depend on $T_{e}$ ]. A simple and quite accurate solution of the rate equations could be found, if we assume that the distribution of outer electrons $(l \geq 3)$ is close to the Boltzmann distribution (this assumption was verified by 

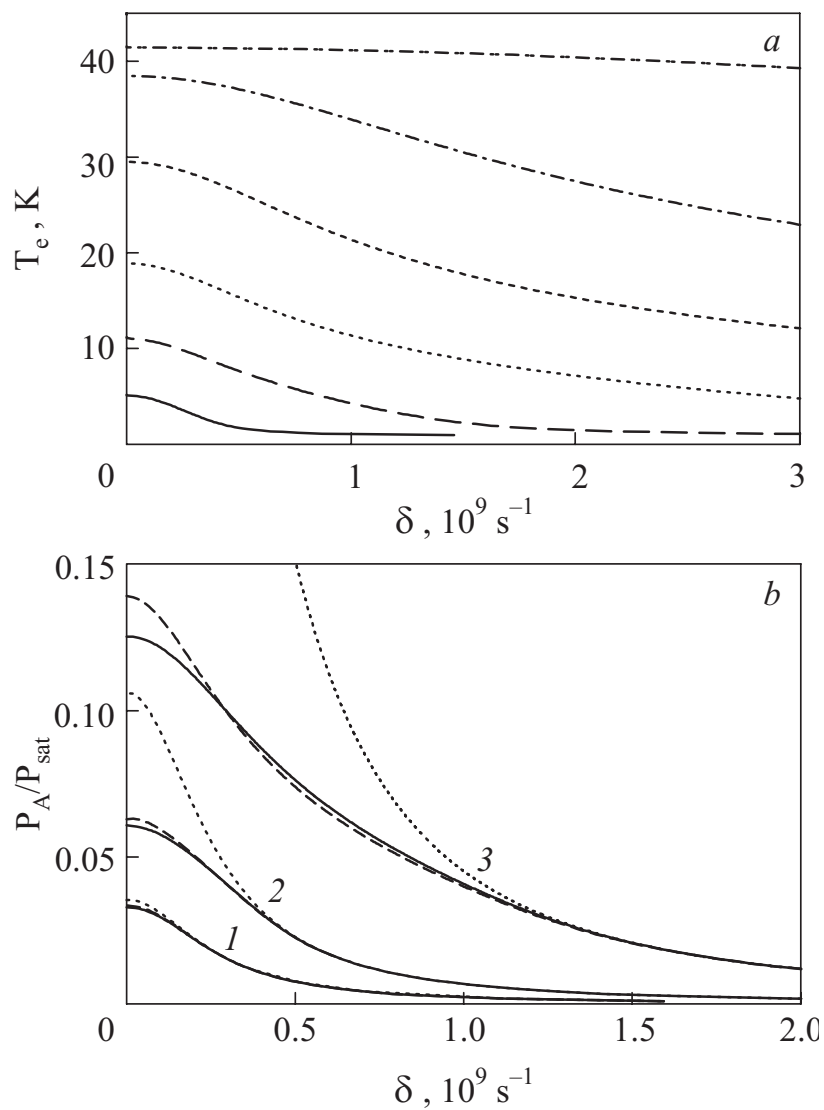

Fig. 1. $T_{e}(a)$ and $P_{\mathrm{A}}(b)$ versus $\delta=\omega-\omega_{0}$ for $T=0.9 \mathrm{~K}$, $E_{\perp} \simeq 106 \mathrm{~V} / \mathrm{cm}$, and different values of $\Omega_{R}$. For $T_{e}(\delta)$, lines correspond (from bottom to top) to $\Omega_{R}$ equal to 1.7, 5.5, 17.3, $54.8,172$, and $10^{3} \mathrm{MHz}$. For $P_{\mathrm{A}}(\delta)$, model of cold SEs (dotted lines), decay heating theory with $\gamma\left(T_{e}\right)=$ const (dashed lines), and decay heating theory with $\gamma\left(T_{e}\right)$ given in Ref. 5 (solid lines) correspond (from bottom to top) to 1 (1), 1.73 (2), and 4.47 (3) $\mathrm{MHz}$ of $\Omega_{R}$.

the exact numerical solution of 400 rate equations). In this case, the problem is reduced to the effective 3-level model which can be solved even analytically, though the final expressions for fractional occupancies $n_{1}\left(T_{e}, r \tau\right)$, $n_{2}\left(T_{e}, r \tau\right)$ and $n_{\text {out }}\left(T_{e}, r \tau\right)=\sum_{l>2} n_{l}$ are cumbersome.

Equation (3) establishes the relation between $T_{e}$ and $r \tau$. Its solution is shown in Fig. 1, $a$ for six distinctive values of Rabi frequency (the in-put power is proportional to $\Omega_{R}^{2}$ ). Even at low excitations, $T_{e}$ substantially exceeds $T \simeq 0.9 \mathrm{~K}$. At high excitations, $T_{e}$ is limited by MW absorption saturation: $T_{e}<T_{e}^{(\max )} \simeq 41.5 \mathrm{~K}$. The typical line $T_{e}(\delta)$ has a resonance form. It is important that the line-width at the half-height of $T_{e}(\delta)$ increases strongly with $\Omega_{R}$ even at $r \tau<<1$. The same is valid for power absorption $P_{\mathrm{A}}(\delta)$ shown in Fig. $1, b$ in units of its limiting value $P_{\text {sat }}$ obtained for $r \tau \rightarrow \infty\left(P_{\text {sat }} \simeq 0.049 \Delta_{21} / 2 \tau\right)$. For the model of cold SEs, the area under the resonance line increases, while the line-width at the half-height remains
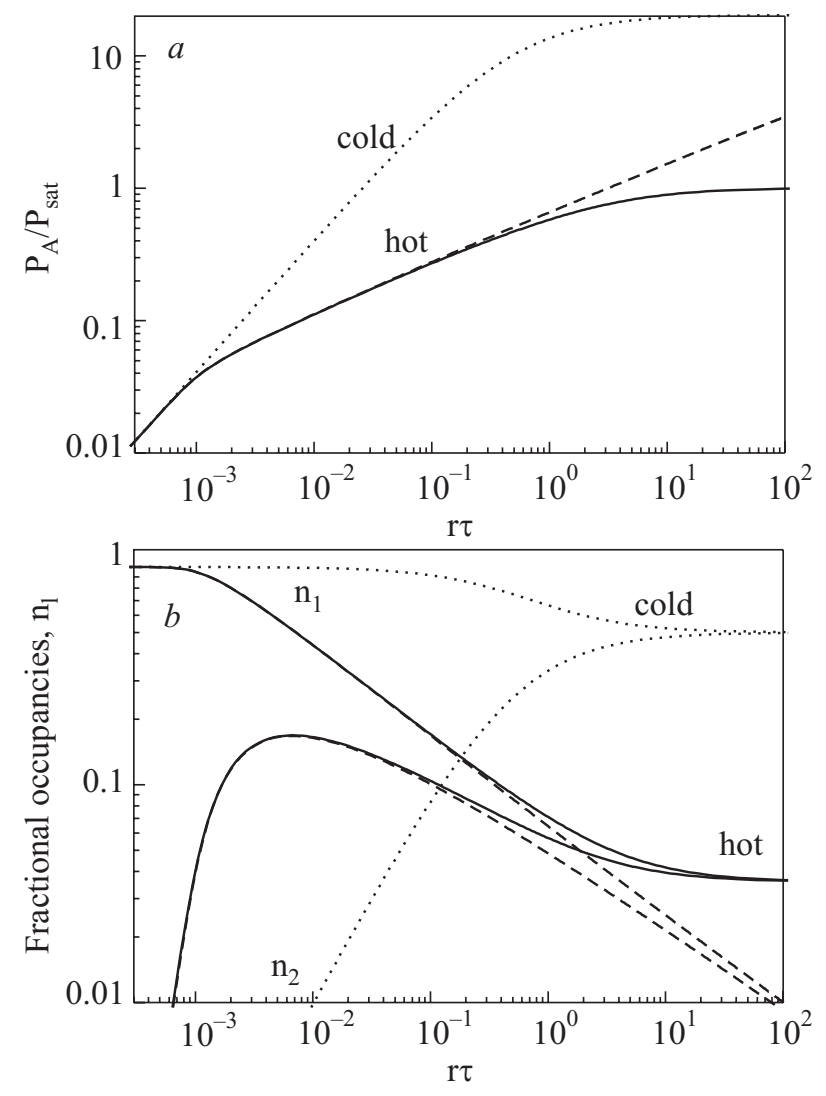

Fig. 2. Power absorption (a) and fractional occupancies (b) versus the excitation parameter $r \tau$ : model of cold SEs (dotted line), decay heating theory (solid line), Boltzmann approximation (dashed line). $T$ and $E_{\perp}$ are the same as in Fig. 1.

approximately the same. Solid and dashed lines represent $P_{\mathrm{A}} / P_{\text {sat }}$ for decay heated electrons (solid line takes into account the increase of $\gamma$ with $T_{e}$ because of scattering to outer levels [5]). The line-width at the half-height of these lines increases strongly with $\Omega_{R}$ even at $r \tau<<1$ $\left(P_{\mathrm{A}}<<P_{\text {sat }}\right)$.

Power absorbed by SEs as a function of the excitation parameter $r \tau$ is shown in Fig. 2, $a$. The dependence $P_{\mathrm{A}}(r \tau)$ expected for cold SEs (dotted line) holds only at $r \tau<7 \cdot 10^{-4}$. At larger values of $r \tau$, the solid curve bends due to the decay heating. Up to about $r \tau \sim 0.1$, this effect can be described also by pure Boltzmann distribution of SEs (dashed line). Still, pure Boltzmann distribution cannot give power saturation up to $r \tau \sim 100$, in spite the fact that $n_{1}-n_{2}$ decreases with $T_{e}$. This can be understood if we consider the left and right sides of Eq. (3) as functions of $T_{e}$ and $r \tau$. For pure Boltzmann distribution, $n_{1}$ and $n_{2}$ depend only on $T_{e}$, and the left side, representing $P_{\mathrm{A}} \tau$, saturates if $T_{e}$ increases with $r \tau$. If $T_{e} \rightarrow$ const, $P_{\mathrm{A}} \tau$ will increase linear with $r \tau$. At the same time, the right side of Eq. (3) do not depend explicitly on $r$, and increases with $T_{e}$ in the actual range of parameters. Therefore, saturation 
requires $T_{e} \rightarrow$ const. This contradiction is eliminated for real occupancies $n_{1}$ and $n_{2}$ which depend explicitly on both $T_{e}$ and $r \tau$.

For $T_{e}(r \tau)$ obtained from Eq. (3), $n_{1}$ and $n_{2}$ are shown in Fig. 2,b. As we can see, strong deviations from the model of cold SEs occur at $r \tau<10^{-3}$. For example, $n_{2}$ increases with $r \tau$ much faster than it is for cold electrons. At higher excitations $\left(r \tau \sim 7 \cdot 10^{-3}\right), n_{2}$ reaches its maximum, and then decreases with $r \tau$. At $r \tau>0.1$, both $n_{1}$ and $n_{2}$ deviate strongly from those given by the Boltzmann approximation (dashed lines), and approach each other much faster when $r \tau \rightarrow \infty$. For decay-heated SEs, the limiting value of $n_{1}$ and $n_{2}$ is approximately an order of magnitude smaller than that obtained previously for $T_{e}=T$.

In conclusion, our study indicates that absorption of MW radiation by electrons on liquid helium is strongly affected by the decay heating, and the simple two-level model fails long before the saturation condition is reached. The decay heating leads to an additional power broadening which can be strong even at low excitations. The important point is that for decay-heated electrons, the absorption saturation appears when the theory is extended beyond the conventional Boltzmann approximation.

This work is partly supported by a Grant-in-Aid for Scientific Research from the Japan Society for the Promotion of Science (JSPS); D.K. thanks JSPS for a postdoctoral fellowship.

1. C.C. Grimes and T.R. Brown, Phys. Rev. Lett. 32, 280 (1974).

2. Yu.P. Monarkha and K. Kono, Two-Dimensional Coulomb Liquids and Solids, Springer-Verlag (2004).

3. E. Collin, W. Bailey, P. Fozooni, P.G. Frayne, P. Glasson, K. Harrabi, M.J. Lea, and G. Papageorgiou, Phys. Rev. Lett. 89, 245301-1 (2002).

4. V.S. Edel'man, Usp. Fiz. Nauk 130, 675 (1980) [Sov. Phys. Usp. 23, 227 (1980)].

5. T. Ando, J. Phys. Soc. Jpn. 44, 765 (1978).

6. M. Saitoh and T. Aoki, J. Phys. Soc. Jpn. 44, 71 (1978).

7. The detailed theoretical description will be given elsewhere. 